\title{
CAPACITY ANALYSIS OF TWO-STAGE PRODUCTION LINES WITH MANY PRODUCTS*
}

\author{
M.B.M. de Koster \\ Department of Industrial Engineering and Management Science, Eindhoven University of Technology, Eindhoven, (The \\ Netherlands)
}

\begin{abstract}
We consider two-stage production lines with an intermediate buffer. A buffer is needed when fluctuations occur. For single-product production lines fluctuations in capacity availability may be caused by random processing times, failures and random repair times. For multiproduct production lines fluctuations are also caused by different processing time ratios for different products and by set up times. We
\end{abstract}

examine whether it is possible to use the results developed for single-product flow lines, where the production units have exponentially distributed life- and repair times, for the multi-product case. As an example the case of a consumer electronics factory is studied.

\section{INTRODUCTION}

In this paper we consider two-stage multiproduct production lines. An example of such a line is the automatic insertion department in the consumer electronics factory described in refs. [1], [2] and [3]. In the automatic insertion department electronic components are inserted mechanically on printed circuit boards. Every printed circuit board requires two different operations. First, certain components are inserted horizontally, then other components are inserted vertically in the same plant. Each component requires either horizontal or vertical insertion.

There are three machines for horizontal insertion and four machines for vertical inser-

*Presented at the Fourth International Working Seminar on Production Economics, Igls, Austria, Feb. 17-21, 1986. tion, separated by a buffer (see Fig. 1).

Essential for the behaviour of the line is that as many as 80 types of panels varying in size and number of components have to be produced. Each panel has it's own processing time and lot-size and many panels require time to set-up. Machine failures may also occur. It is clear that we need intermediate storage facility and a production control rule for using this facility, to buffer against temporary imbalances between the insertion machines.

In Table 1 some characteristics of the line are given in terms of components.

Vertical insertion speeds in Table 1 are expressed in terms of buffered components and not in terms of vertical component insertions. A speed of $180 \mathrm{comp} . / \mathrm{min}$. for a vertical inserter does not mean that 180 components are inserted vertically per minute, but that 180 


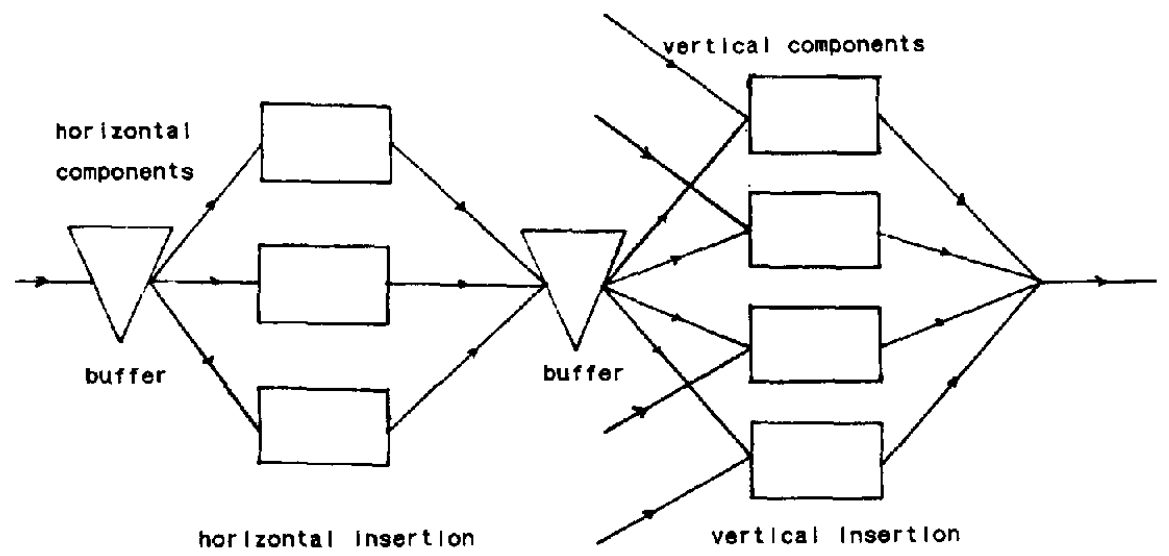

Fig. 1. The automatic insertion department.

TABLE 1

Parameters of horizontal and vertical insertiơn machines

\begin{tabular}{llllll}
\hline & $\begin{array}{l}\text { Machine speeds } \\
\text { (comp./min.) }\end{array}$ & $\begin{array}{l}\text { Change-over } \\
\text { time } \\
(\text { min. })\end{array}$ & $\begin{array}{l}\text { Down-time } \\
\text { (min.) }\end{array}$ & $\begin{array}{l}\text { Total } \\
\text { down-time }\end{array}$ & $\begin{array}{l}\text { Batch-size } \\
(\text { comp.) }\end{array}$ \\
\hline Horizontal ins. & $3 \times 230$ & 8.3 & 12 & $10 \%$ & 8000 \\
Vertical ins. & $4 \times 180$ & 10.0 & 11 & $14 \%$ & \\
\hline
\end{tabular}

already horizontally inserted components are processed per minute. We assume that always enough horizontal components are present to be inserted horizontally and that always enough vertical components are present to be inserted vertically. The average cycle times of the inserters are small compared to average downtimes and runtimes. This implies that we may use a continuous time model with production rates instead of service times.

The numbers in Table 1 are averages. Batch sizes are in fact random. Each batch is processed by only one horizontal and one vertical inserter. If one of the three horizontal inserters fails or is down for a set-up the horizontal insertion-speed diminishes with 230 comp./min. Hence the horizontal insertion production unit may work at 4 different speeds (690, 460, 230 and 0 comp./min.).

In the factory the orders in the whole production line, of which the insertion depart- ment is but a small part, are controlled by the Goods Flow Control department (GFC). GFC releases orders to the departments dependent on the work-in-process norms and the actual work in process. In order to determine this work-in-process norm for the insertion department, the throughput and leadtime of the department have to be determined as a function of the work-in-process. Work-in-process in the line consists of all panels in the insertion machines, in the buffer and in the queue of released panels in front of horizontal insertion. We call this queue also a buffer. We assume however, that the work-in-process level equals the number of panels (or components) in the buffers, only. This is justified since the number of components in the insertion machines is approximately constant. If the work-in-process is kept at level $K$, then the insertion line is equivalent, as far as the throughput is concerned, with a two-stage line 
consisting of horizontal and vertical insertion and an intermediate buffer of physical capacity $K$.

The leadtime of the insertion line for an individual batch always consists of horizontal and vertical processing times plus usually some waiting time if another batch is being processed or if the right panels are not available and is therefore more or less fixed. Hence the only remaining problem is the dependence of the throughput on the buffer capacity and our objective is therefore to analyse the two-stage insertion line in order to establish this relationship.

In section 2 a model will be outlined by which the insertion department can be analysed. The model is typical for two-stage multiproduct flowlines in the sense that machine imbalances due to different products requiring different capacity profiles over the subsequent work stations are incorporated.

It will be shown that the complicated flow lines of the above type can be approximated by simple exponential models. Exponential models have the advantage that the throughput can be calculated easily.

In section 3 some numerical results are presented. In section 4 we return to the original case and draw some conclusions.

\section{THE MODEL}

The model we use for the description of the case mentioned in the introduction is sketched in Fig. 2.

Production unit 1 (PU1) in the line of Fig. 2 works at speeds $\nu_{1}, \ldots, \nu_{\mathrm{n}}$ (not necessarily different). PU1 works at speed $\nu_{i}$ during an exponentially distributed interval (parameter $\lambda_{\mathrm{i}}$ ). After a period with speed $\nu_{\mathrm{i}}$ a transition takes place with probability $p_{\mathrm{ij}}$ to a state with speed $\nu_{j}$. The matrix $P=\left(p_{i j}\right)$ is Markov, that is $p_{\mathrm{ij}} \geqq 0$ and $\sum_{j=1}^{n} p_{\mathrm{ij}}=1$, for all i. Production unit PU2 is similar to PU1, with speeds $\omega_{1}, \ldots, \omega_{\mathrm{k}}$, exponential rates $\mu_{1}, \ldots, \mu_{\mathrm{k}}$ and transition matrix $Q$.
The buffer capacity is $K$.

Note that, by choosing some $\nu_{\mathrm{i}}$ 's equal, it is possible to generate arbitrary phase-type distributions for the intervals that a production unit is working at a certain speed. Several interpretations are possible. We may interprete $\mu_{i} / \lambda_{i}$ as the average lot-size of $i$ and by choosing $\mu_{\mathrm{n}}=0$ and $p_{\mathrm{in}}=1$, for all $\mathrm{i} \neq \mathrm{n}$, and $p_{\mathrm{nn}}=0$ a change-over time can be represented.

Since the buffer $(B)$ has finite capacity, blocking of the PU's may occur. If PU2 is not working and $B$ is full then PU1 is blocked. If PU2 is working at speed $\omega_{\mathrm{i}}, B$ is full and PU1 is working at speed $\nu_{\mathrm{j}}$ with $\nu_{\mathrm{j}}>\omega_{\mathrm{i}}$, then PU1 is slowed down to speed $\omega_{\mathrm{i}}$. If $B$ is empty then PU2 may similarly be slowed down (or forced down if PU1 is down). We suppose that PU1 is never starved by lack of products and PU2 is never blocked by lack of finished product storage capacity. We take the goodsflow to be continuous. The throughput of this line is denoted by $v(K)$ and can be calculated by using a method similar to the one developed in [4]. If PU1 has $n$ different states and PU2 has $k$ different states, then determining $v(K)$ requires the solution of a system of $k \cdot n$ first order linear differential equations. This may give computational problems if $k \cdot n$ is large.

In this paper it will be proven that complicated two-stage production lines, as sketched in Fig. 2, can be approximated by simpler exponential lines.

In order to make such an approximation we only have to know five parameters of the original line. These parameters are first and second moments of the buffer increase and buffer decrease and the expected length of an increase period plus the decrease period.

The approximation is based on the idea that the buffer content behaves as a random walk with barriers and that the throughput of the line is determined completely by the random walk and the relation of the random walk with time. In fact there are only four relevant parameters since it will be shown that for two different production lines with the same first and sec- 


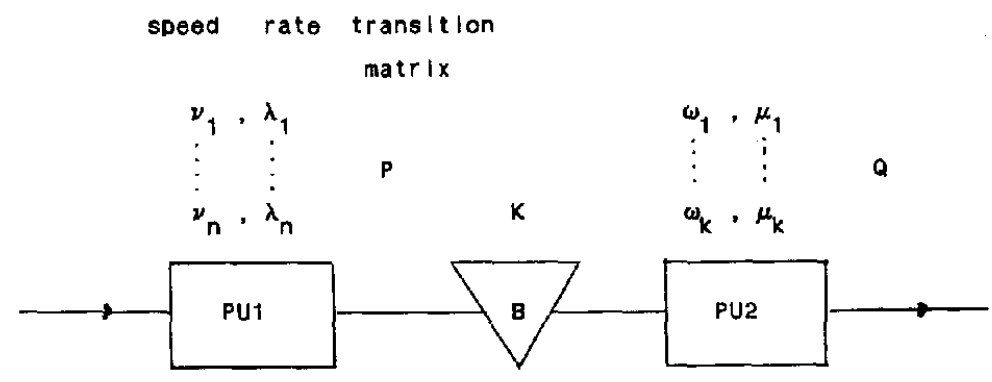

Fig. 2. Two-stage multi-product production line.

ond moments of buffer increase and buffer decrease the average loss in production per unit of time (that is, the difference of the line production rate with infinite and with finite buffer) times the expected length of an increase plus decrease period is constant. Or, in formula

$$
(v(\infty)-v(K))(E L+E M)=C
$$

where $v(K)$ is the line throughput with buffer capacity $K, L$ and $M$ are the lengths of a period of buffer increase and decrease, respectively, and $E$ is the expectation operator. In Fig. 3 we have sketched a realisation of the buffer content as a function of time for a certain two-stage line of the type of Fig. 2 for a finite buffer as well as for an infinite buffer. Furthermore the buffer increase, $T$, and buffer decrease, $S$, are shown. A pair $(a, b)$ denotes that machine 1 runs at rate $a$, machine 2 at rate $b$. The throughput is obtained as the quotient of the output of machine 2 and the elapsed time.

In order to test the hypothesis (2.1) we consider a model where PU2 has only two different speeds, that is $k=2$, such that $\omega_{2}=0, \omega_{1}=$ : $\omega, Q=\left(\begin{array}{ll}0 & 1 \\ 1 & 0\end{array}\right)$. We also suppose there is an $m<n$ such that $\nu_{1}>\omega, \ldots, \nu_{\mathrm{m}}>\omega$ and $\nu_{\mathrm{m}+1}<\omega, \ldots \nu_{\mathrm{n}}<\omega$. However, the analysis for arbitrary $k, \omega_{\mathrm{i}}, \nu_{\mathrm{j}}$ and $Q$ is completely analogous. This special case of the line is denoted line $I$ and sketched in Fig. 4.

For the line of Fig. 4 we calculate the behaviour of the buffer content. Let $T$ and $S$ be the increase and decrease of the buffer content, respectively, and $L$ and $M$ the length of such an increase and decrease period, then we cal- culate first and second moments of $T$ and $S$, and first moments of $L$ and $M$. We do this by solving linear equations similar to those given in [5]. To obtain $E T^{2}\left(E S^{2}\right)$ we first have to calculate $E T(E S)$. The calculation of these moments is computationally much less involved than the calculation of the throughput since here a system of at most $2 \cdot n$ linear equations has to be solved.

Let $c_{\mathrm{T}}$ be the coefficient of variation of $T$, that is,

$c_{\mathrm{T}}=\frac{\sqrt{E T^{2}-(E T)^{2}}}{E T}$

and $c_{\mathrm{S}}$ the coefficient of variation of $S$. We approximate line I by line II, sketched in Fig. 5 , in such a way that $E T, E T^{2}, E S$ and $E S^{2}$ remain invariant and we investigate the difference in throughput.

In line II of Fig. 5 PU2 is completely reliable with production rate 1 . PU1 only has two different speeds, $w_{1}>1$ and $w_{2}<1$. For the $w_{1}$ interval and $w_{2}$-interval we use distributions of one of the following two types. Dependent on whether or not $c_{\mathrm{T}} \geqq 1\left(c_{\mathrm{S}} \geqq 1\right)$ a $w_{1}$-interval ( $w_{2}-$ interval) has a two-stage hyperexponential- or two-stage Cox-distribution as sketched in Fig. 6, with branching probability $p(q)$. Hyperexponential distributions always have a coefficient of variation $>1$. Cox-distributions may have a coefficient of variation $>1$ or $<1$. Each stage in the hyperexponential- and Coxdistribution of Fig. 6 has an exponential distribution.

Note that line II of Fig. 5 is a special case of 


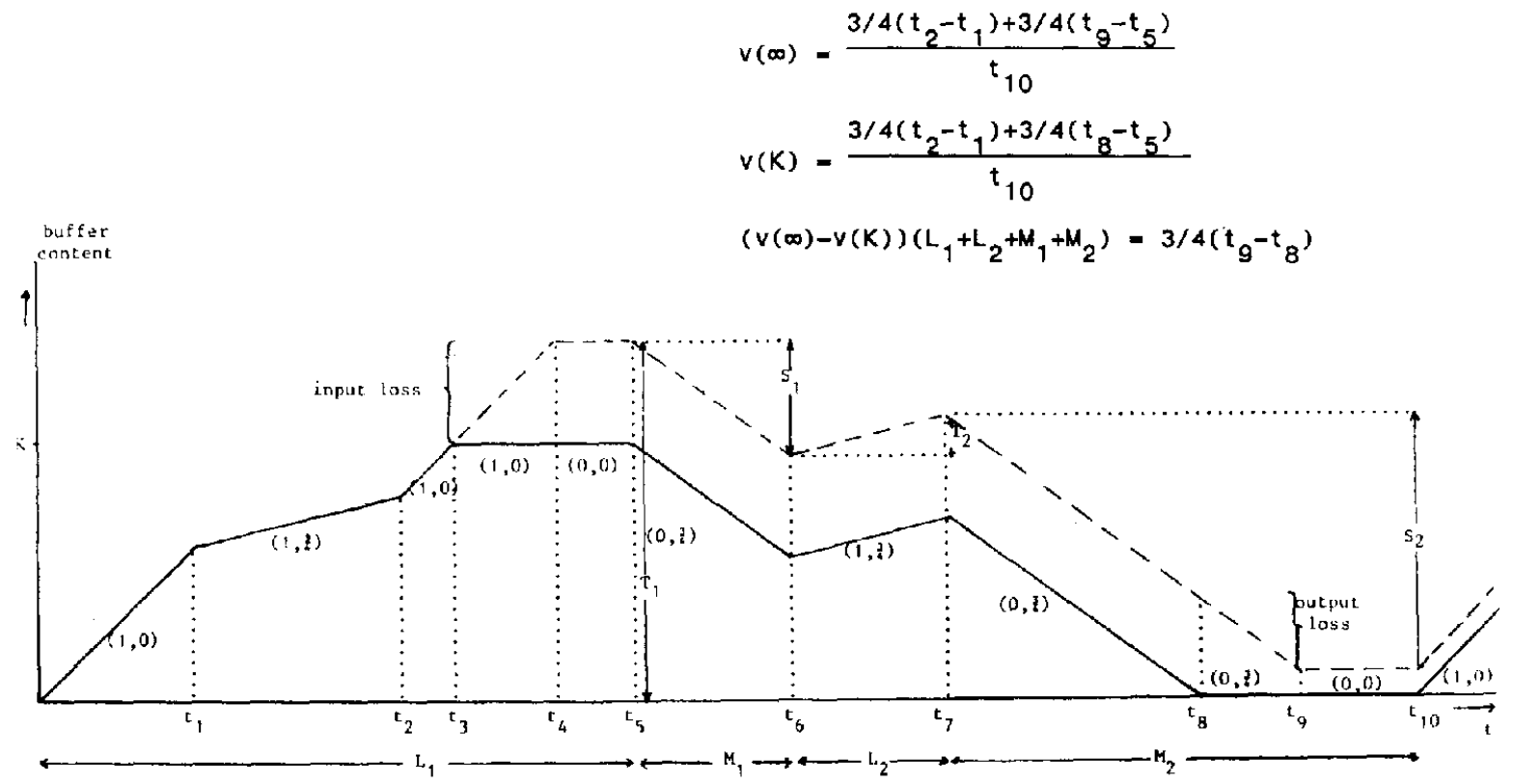

Fig. 3. Behaviour of the buffer content for a certain two-stage line with buffer capacity $K(\ldots$ ) and infinite buffer (---). At time $t_{3}$ the buffer becomes full, which leads to loss in input because PU1 is still operating. At time $t_{8}$ the buffer becomes empty, which leads to loss in output from PU2.

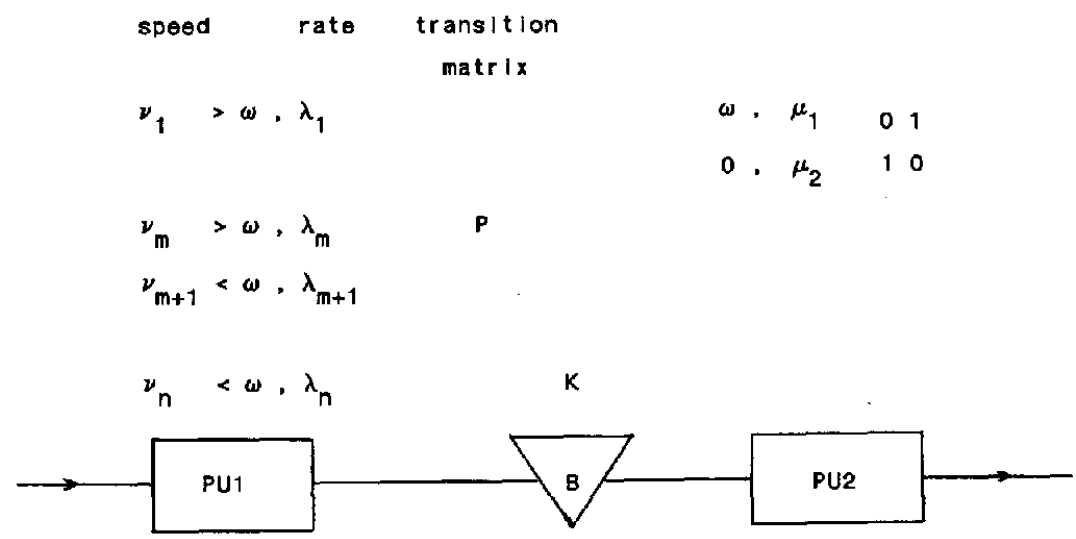

Fig. 4. "Line I", special case of the line of Fig. 2.

line I, with $n=4, m=2, \lambda_{\mathrm{i}}=\rho_{\mathrm{i}}(\mathrm{i}=1,2), \lambda_{\mathrm{j}+2}=\eta_{\mathrm{j}}$ $(j=1,2), \mu_{1}=0$ and $\nu_{1}=\nu_{2}=w_{1}, \nu_{3}=\nu_{4}=w_{2}$, $\omega=1$. If an interval of speed $w_{1}$ has a hyperexponential distribution with branching probability $p$ and an interval of speed $w_{2}$ has a Coxdistribution with branching probability $q$ then the transition matrix $R$ has the following form

$R=\left[\begin{array}{llll}0 & 0 & 1 & 0 \\ 0 & 0 & 1 & 0 \\ (1-q) p & (1-q)(1-p) & 0 & q \\ p & 1-p & 0 & 0\end{array}\right]$

For line II the buffer quantities $E T^{n}, E S^{n}$, $E L$ and $E M$ can be calculated easily for all $n \geqq 1$. 


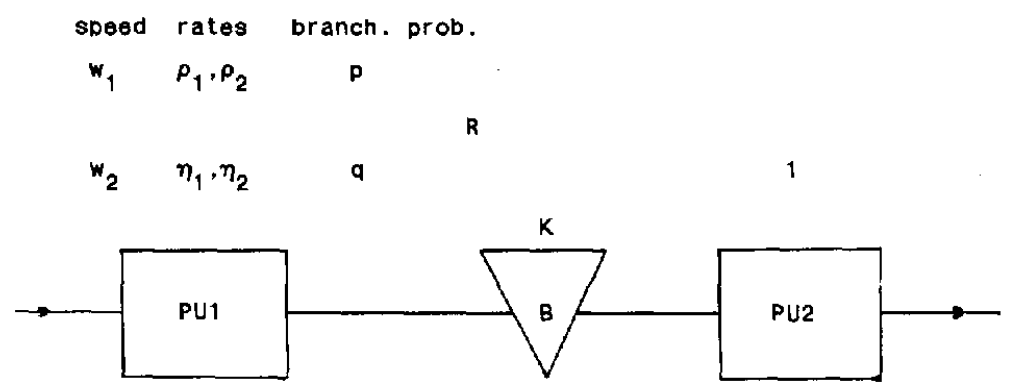

Fig. 5. "Line II", approximating line I of Fig. 2.

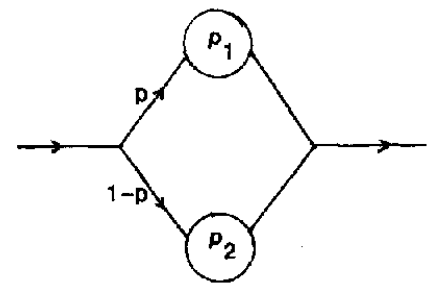

hyperexponentlal distribution

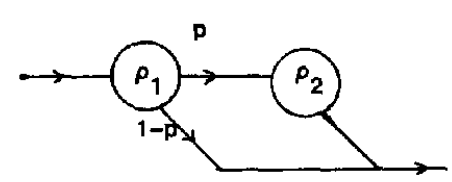

cox-distribution

Fig. 6. Two-stage hyperexponential- and Cox-distributions.

If an interval of speed $w_{1}$ has a hyperexponential distribution as sketched in Fig. 6, we have

$$
\begin{aligned}
& E T^{n}=n !\left(w_{1}-1\right)^{n}\left(\frac{p}{\rho_{2}{ }^{n}}+\frac{1-p}{\rho_{2}{ }^{n}}\right), n \geqq 1 \\
& E L=\frac{p}{\rho_{1}}+\frac{1-p}{\rho_{2}}
\end{aligned}
$$

If a $w_{1}$-interval has a two-stage Cox-distribution we have

$$
\begin{aligned}
& E T^{\mathrm{n}}=n !\left(w_{1}-1\right)^{n}\left(\frac{1}{\rho_{1} n}\right. \\
& \left.\quad+p \sum_{k=1}^{n}\left(\frac{1}{\rho_{2}}\right)^{k}\left(\frac{1}{\rho_{1}}\right)^{n-k}\right), n \geqq 1 \\
& E L=\frac{1}{\rho_{1}}+\frac{p}{\rho_{2}}
\end{aligned}
$$

If a $w_{2}$-interval has a hyperexponential- or Cox-distribution, then $E S^{\mathrm{n}}$ and $E M$ are obtained from formulas (2.3) or formulas (2.4), respectively by replacing $w_{1}-1$ by $1-w_{2}, p$ by $q$ and $\rho_{\mathrm{i}}$ by $\eta_{\mathrm{i}}(\mathrm{i}=1,2)$.
Now suppose $E T, c_{\mathrm{T}}, E S$ and $c_{\mathrm{s}}$ of line I are given. We want to determine $w_{\mathrm{i}}, \rho_{\mathrm{i}}, \eta_{\mathrm{i}}, p$ and $q$ such that the first and second moments of buffer increase and decrease of line II, as given in formulas (2.3) and (2.4) equal the corresponding moments of line I. If $c_{\mathrm{T}} \geqq 1$ then, for arbitrary $w_{1}>1$ and $p$ the rates of the hyperexponential $w_{1}$-interval determined by

$$
\begin{aligned}
\rho_{1}=\frac{w_{1}-1}{E T} & {\left[1+\left(\frac{1-p}{p} \frac{c_{\mathrm{T}}^{2}-1}{2}\right)^{1 / 2}\right]^{-1} } \\
\rho_{2}=\frac{w_{1}-1}{E T} & {[1} \\
& \left.-\left(\frac{p}{1-p} \frac{c_{\mathrm{T}}^{2}-1}{2}\right)^{1 / 2}\right]^{-1}
\end{aligned}
$$

make first and second moments of buffer increase equal (see [6]). The choice of $p$ is arbitrary under the condition that $\rho_{2}>0$. Since for the choice of these parameters there are only two conditions. Other solutions are also possible. Now suppose $c_{\mathrm{T}}<1$ then, for arbitrary $w_{1}>1$ the parameters of the Cox-distribution may be determined as follows (see [7]) 
$p=1-\frac{2 c_{\mathrm{T}}^{2}-\left(2-2 c_{\mathrm{T}}^{2}\right)^{1 / 2}}{c_{\mathrm{T}}^{2}+1}$

$\rho_{1}=\rho_{2}=\frac{w_{1}-1}{E T}(1+p)$

This choice is also not unique. If $c_{\mathrm{S}} \geqq 1$ or $c_{\mathrm{S}}<1$, $q, \eta_{1}, \eta_{2}$ are determined similarly, in order to make first and second moments of buffer decrease equal.

Since we still have freedom left in the choice of $w_{1}$ and $w_{2}$ this freedom could be used to make $E L_{\mathrm{I}}+E M_{\mathrm{I}}$ and $E L_{\mathrm{II}}+E M_{\mathrm{II}}$ equal. Now let "line $a$ " and "line $b$ " be two different lines II, with hyperexponential $w_{1}$-interval and $w_{2}$ interval. From formulas (2.3) and the analogous form with $\eta, q$ and $w_{2}$ instead of $\rho, p$ and $w_{1}$, it follows that if

$$
\begin{aligned}
& \frac{\rho_{\mathrm{ia}}}{w_{1 \mathrm{a}}-1}=\frac{\rho_{\mathrm{ib}}}{w_{\mathrm{b}}-1} \quad i=1,2 \\
& \frac{\eta_{\mathrm{ia}}}{1-w_{2 \mathrm{a}}}=\frac{\eta_{\mathrm{ib}}}{1-w_{2 \mathrm{~b}}} \quad i=1,2 \\
& p_{\mathrm{a}}=p_{\mathrm{b}} \\
& q_{\mathrm{a}}=q_{\mathrm{b}}
\end{aligned}
$$

we have $E T_{\mathrm{a}}^{\mathrm{n}}=E T_{\mathrm{b}}^{\mathrm{n}}$ and $E S_{\mathrm{a}}^{\mathrm{n}}=E S_{\mathrm{b}}^{\mathrm{n}}$, for all $n$. In this case relation (2.1) will hold exactly. That is,

$$
\begin{aligned}
& \left(v_{\mathrm{IIa}}(\infty)-v_{\mathrm{IIa}}(K)\right)\left(E L_{\mathrm{a}}+E M_{\mathrm{a}}\right) \\
& \quad=\left(v_{\mathrm{Ibb}}(\infty)-v_{\mathrm{IIb}}(K)\right)\left(E L_{\mathrm{b}}+E M_{\mathrm{b}}\right)
\end{aligned}
$$

In [5] relation (2.8) is proven for the case where $\rho_{1}=\rho_{2}, \eta_{1}=\eta_{2}$ and $w_{2}=0$. Relation (2.8) implies that the choice of $w_{1}$ and $w_{2}$ has no effect on the quality of the approximation, as long as condition (2.7) is satisfied. Therefore we do not use this freedom to make $E L_{\mathrm{I}}+E M_{\mathrm{I}}$ and $E L_{\mathrm{II}}+E M_{\mathrm{II}}$ equal.

\section{NUMERICAL RESULTS}

In this section we compare the throughputs of lines I and II, with buffer capacity $K$, under the condition that the parameters of line II are chosen such that first and second moments of buffer increase and buffer decrease are equal. In order to take the frequency of buffer increase and buffer decrease into account, rather than comparing $v_{\mathrm{I}}(K)$ and $v_{\mathrm{II}}(K)$, we have to compare $v_{1}(K)$ and

$$
\begin{aligned}
& \hat{v}_{\mathrm{I}}(K):=v_{\mathrm{I}}(\infty)-\left(v_{\mathrm{II}}(\infty)\right. \\
& \left.-v_{\mathrm{II}}(K)\right)\left(E L_{\mathrm{II}}+E M_{\mathrm{II}}\right) /\left(E L_{\mathrm{I}}+E M_{\mathrm{I}}\right)
\end{aligned}
$$

Relative errors are measured in the following way

$\epsilon(K)=\frac{v_{\mathrm{I}}(K)-\hat{v}_{\mathrm{I}}(K)}{v_{\mathrm{I}}(\infty)-v_{\mathrm{I}}(K)} \times 100 \%$

The reason for using this (strong) definition of relative error is that we want to measure the relative error in the loss of production due to a finite buffer rather than in the production rate itself.

The approximation method is now tested on five different examples of line I. In these lines PU1 has 2, 5, 3, 5 and 7 states, respectively. The numbers of states in these lines vary from 4 to 14 and hence the influence of increasing complexity on the quality of the approximation can be studied. For the last three lines $E T$, $E S$ and $E L+E M$ are fixed, but $c_{\mathrm{T}}$ and $c_{\mathrm{S}}$ decrease with the number of states. In Table 2 the parameters of line I and the approximating line II for the first case are presented.

PU1 in line I can be interpreted as an unreliable PU with failure rate $\lambda_{1}$ and repair rate $\lambda_{2}$. In Table 3 relative errors of the approximation are given.

Relative errors in Table 3 are small. In Table 4 a second line I and an approximating line II are listed. Note that PU2 in line I is perfect. In Table 5 relative errors of the approximation are given.

Relative errors in Table 5 are large, but this is not serious since $v_{1}(K)-\hat{v}_{1}(K)$ is small anyway. However, in view of these large errors we also used a three-moment approximation of line I. Third moments of buffer increase and 
TABLE 2

Parameters of line I and the approximating line II

\begin{tabular}{lllllllll}
\hline$n=2$ & Prod. rate & Life rate & Trans. matrix & $E L+E M$ & $E T$ & $c_{\mathbf{T}}$ & $E S$ & $c_{\text {S }}$ \\
\hline Line I & $\nu_{1}=1.0$ & $\lambda_{1}=0.01$ & & 116.959 & 15.789 & 1.357 & 10.526 & 1.0 \\
& $\nu_{2}=0$ & $\lambda_{2}=0.09$ & $P=\left(\begin{array}{ll}0 & b \\
l & b\end{array}\right)$ & & & & & \\
& $\omega=1.05$ & $\mu_{1}=0.01$ & & & & & & \\
& & $\mu_{2}=0.09$ & & & & & \\
\hline Line II & $w_{1}=1.1$ & $\rho_{1}=0.004$ & $p=0.5$ & 169.591 & & & \\
& $w_{2}=0.018$ & & & & & & \\
& $w_{2}=0.1$ & $\eta_{1}=0.086$ & $q=0.5$ & & & & & \\
& & $\eta_{2}=0.086$ & & & & & & \\
\hline
\end{tabular}

TABLE 3

Relative errors in approximation of line I of Table 2

\begin{tabular}{rllc}
\hline$K$ & $v_{\mathrm{I}}(K)$ & $\hat{v}_{\mathrm{I}}(K)$ & $\epsilon$ \\
\hline 0 & 0.81 & 0.81 & 0 \\
5 & 0.8367 & 0.8386 & -3.00 \\
10 & 0.8511 & 0.8537 & -5.32 \\
20 & 0.8682 & 0.8702 & -6.29 \\
30 & 0.8778 & 0.8794 & -7.21 \\
40 & 0.8840 & 0.8851 & -6.88 \\
$\infty$ & 0.9 & 0.9 & - \\
\hline
\end{tabular}

buffer decrease can be obtained by using known first and second moments as first moments are used to calculate second moments.

In [8] a method is presented for approximating a distribution with known first three moments and coefficient of variation $>1$ by a two-stage Cox-distribution with the same first three moments. Using results of [8] it follows that it is possible to keep the first three moments of buffer increase in line II equal to the first three moments of buffer increase in line $\mathrm{I}$, by taking in case $c_{\mathrm{T}}>1$, for $w_{1}>1$ arbitrary,

$\rho_{1}=\left(X+\sqrt{X^{2}-4 Y}\right) / 2$

$\rho_{2}=X-\rho_{1}$

$p=\frac{\rho_{2}}{\rho_{1}}\left(m_{1} \rho_{1}-1\right)$

where

$Y=\left(6 m_{1}-3 m_{2} / m_{1}\right) /\left[\left(3 m_{2}^{2} / 2 m_{1}\right)-m_{3}\right]$

TABLE 4

Parameters of line I and approximating line II

\begin{tabular}{|c|c|c|c|c|c|c|c|c|c|c|}
\hline$n=5$ & Prod. rate & Life rate & Trans. matrix & & & $E L+E M$ & $E T$ & $c_{\mathbf{T}}$ & $E S$ & $c_{\mathbf{s}}$ \\
\hline Line I & $\begin{array}{l}\nu_{1}=1.5 \\
\nu_{2}=1.1 \\
\nu_{3}=1.5 \\
\nu_{4}=0.9 \\
\nu_{5}=0.01 \\
\omega=1\end{array}$ & $\begin{array}{l}\lambda_{1}=0.2 \\
\lambda_{2}=0.2 \\
\lambda_{3}=0.01 \\
\lambda_{4}=0.01 \\
\lambda_{5}=0.02 \\
\mu_{1}=0\end{array}$ & $P=\left[\begin{array}{ll}0 & 0 \\
0 & 0 \\
0 & 0 \\
0 & 0.1 \\
0.2 & 0.3\end{array}\right.$ & $\begin{array}{l}0 \\
0 \\
0 \\
0.9 \\
0.5\end{array}$ & $\left.\begin{array}{ll}0.2 & 0.8 \\
0.9 & 0.1 \\
0.9 & 0.1 \\
0 & 0 \\
0 & 0\end{array}\right]$ & 180.27 & 4.387 & 1.11 & 14.59 & 1.58 \\
\hline Line II & $\begin{array}{l}w_{1}=1.2 \\
w_{2}=0.8\end{array}$ & $\begin{array}{l}p_{1}=0.034 \\
p_{2}=0.069 \\
\eta_{1}=0.007 \\
\eta_{2}=0.104\end{array}$ & $\begin{array}{l}p=0.5 \\
q=0.5\end{array}$ & & & 94.901 & & & & \\
\hline
\end{tabular}


TABLE 5

Relative errors in approximation of line I of Table 4

\begin{tabular}{rllc}
\hline$K$ & $v_{\mathrm{I}}(K)$ & $\hat{v}_{\mathrm{I}}(K)$ & $\epsilon$ \\
\hline 0 & 0.9190 & 0.9190 & 0 \\
5 & 0.9333 & 0.9320 & 13.24 \\
10 & 0.9385 & 0.9368 & 34.38 \\
20 & 0.9420 & 0.9409 & 83.11 \\
30 & 0.9430 & 0.9424 & 146.82 \\
40 & 0.9433 & 0.9430 & 233.06 \\
$\infty$ & 0.9434 & 0.9434 & - \\
\hline
\end{tabular}

$X=1 / m_{1}+m_{2} Y / 2 m_{1}$

$m_{1}=\frac{E T^{\mathrm{i}}}{\left(w_{1}-1\right)^{\mathrm{i}}}, \quad i=1,2,3$

However, note that a three-moment approximation by a two-stage Cox-distribution is only possible if $3 m_{2}^{2}<2 m_{1} m_{3}$ and if the coefficient of variation $>1$.

If $c_{\mathrm{S}}>1 \eta_{1}, \eta_{2}$ and $q$ are determined similarly for arbitrary $w_{2}<1$. Instead of $w_{1}-1$ we have to take $1-w_{2}$. Parameters of the line II are now chosen by a three-moment method. Relative errors of this approximation are given in Table 6.

The relative errors in Table 6 are much smaller than the relative errors in Table 5 . However, the three-moment method does not perform better in general than the two-moment method as the following examples will show.

We now consider three different production lines I, with 3,5 or 7 states for PU1, respectively (see Table 7). For all cases we have
$E L+E M=111.11, E T=21.51, E S=18.90$ and PU1 has average machine speed 0.9235 . For PU2 we have for all cases $\omega=1.0, \mu_{1}=0.01$ and $\mu_{2}=0.09$. The major difference between the three cases is that for increasing $n, c_{\mathrm{T}}$ and $c_{\mathrm{S}}$ decrease, that is the production process becomes more stable.

In Table 7 the approximating lines II are also given. For case 1 we have two approximating lines, one based on a two-moment approximation ("line $a$ ") and one based on a threemoment approximation ("line $b$ ").

Numerical results of the approximations are given in Table 8.

In Table 8 we see that for case 1 approximation by line $b$ keeping equal the first three moments does not perform better than the approximation by line $a$, with only first and second moments kept equal. Note that for increasing $n, v_{\mathrm{I}}(K)$ increases for $0<K<\infty$. This fact is due to the more stable situation we get for increasing $n$.

\section{CONCLUSIONS}

In the previous section it has been shown that two-stage production lines with complicated PU's can be very well approximated by simple exponential lines, provided we can keep first and second moments of buffer increase and buffer decrease invariant. Higher moments of buffer increase and buffer decrease do not play a significant role.

We now return to the case mentioned in the introduction and the problem of determining

TABLE 6

Approximations of line I by three-moment method

\begin{tabular}{rccccccc}
\hline$K$ & $V_{\mathrm{I}}(K)$ & $\hat{V}_{\mathrm{I}}(K)$ & $\epsilon$ & Mach. speed & Life rate & Trans. prob. & $E L+E M$ \\
\hline 0 & 0.9190 & 0.9190 & 0 & $w_{1}=1.051$ & $\rho_{1}=0.084$ & $p=0.757$ & 180.267 \\
5 & 0.9333 & 0.9333 & -0.41 & & $\rho_{2}=0.010$ & \\
10 & 0.9385 & 0.9386 & -1.47 & $w_{2}=0.845$ & $\eta_{1}=0.015$ & $q=0.093$ \\
20 & 0.9420 & 0.9421 & -4.50 & & $\eta_{2}=0.003$ & \\
30 & 0.9430 & 0.9430 & -7.81 & & & \\
40 & 0.9433 & 0.9433 & -11.01 & & & \\
$\infty$ & 0.9434 & 0.9434 & - & & & \\
\hline
\end{tabular}


184

TABLE 7

Three lines I with approximating lines II

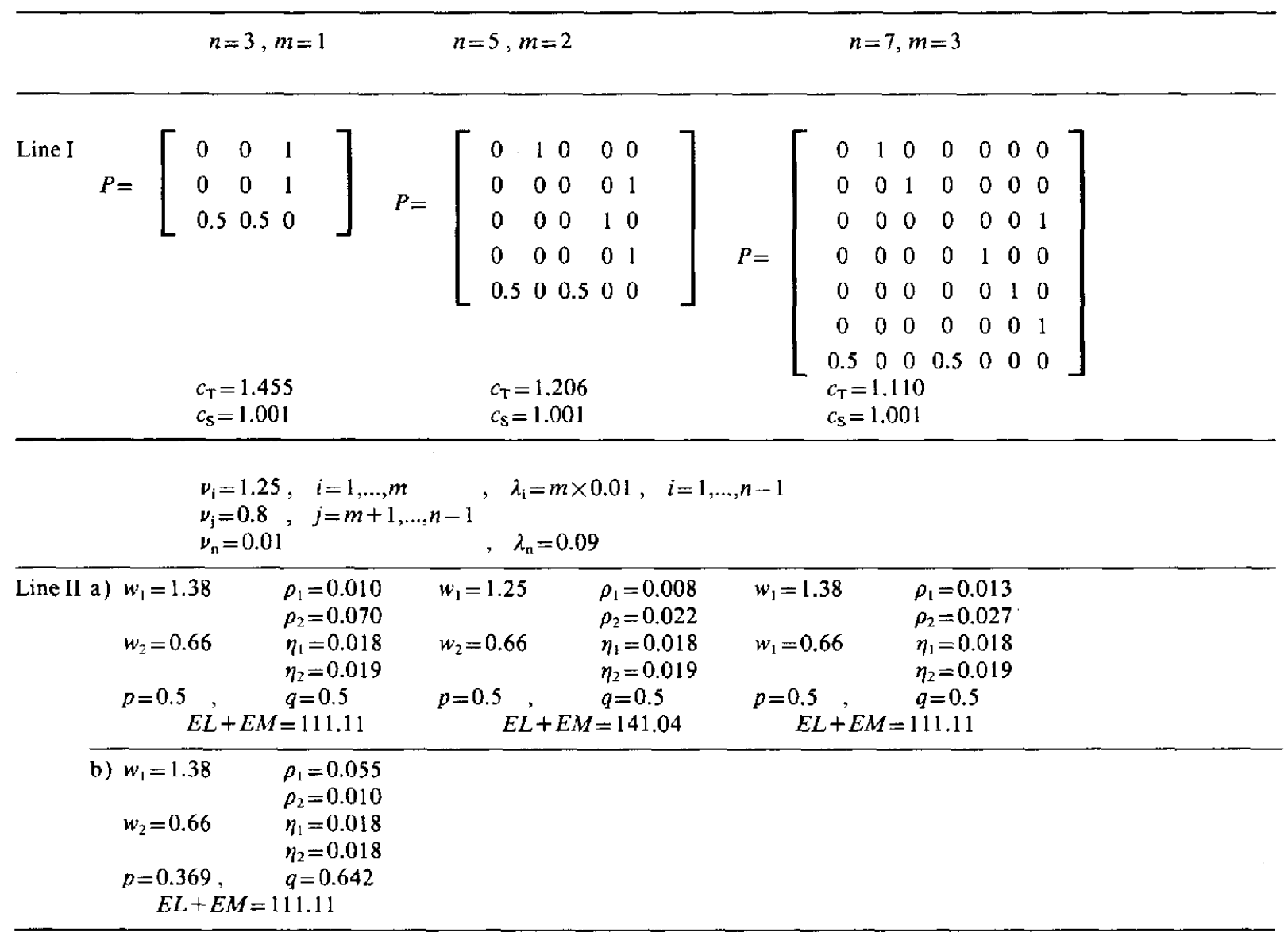

TABLE 8

Relative errors in approximations of lines I of Table 7

\begin{tabular}{|c|c|c|c|c|c|c|c|c|c|c|c|}
\hline$K$ & $V_{1}(K)$ & $\hat{V}_{\mathrm{Ia}}(K)$ & $\epsilon$ & $\hat{V}_{1 \mathrm{~b}}(K)$ & $\epsilon$ & $V_{1}(K)$ & $\hat{V}_{1}(K)$ & $\epsilon$ & $V_{1}(K)$ & $\hat{V}_{\mathbf{I}}(K)$ & $\epsilon$ \\
\hline 0 & 0.7299 & 0.7299 & 0 & 0.7299 & 0 & 0.7299 & 0.7299 & 0 & 0.7299 & 0.7299 & 0 \\
\hline 5 & 0.7625 & 0.7626 & -0.1 & 0.7633 & -0.6 & 0.7635 & 0.7651 & -1.1 & 0.7637 & 0.7655 & -1.3 \\
\hline 10 & 0.7828 & 0.7823 & 0.4 & 0.7837 & -0.8 & 0.7853 & 0.7878 & -2.2 & 0.7860 & 0.7890 & -2.6 \\
\hline 20 & 0.8078 & 0.8073 & 0.5 & 0.8097 & -2.1 & 0.8123 & 0.8161 & -4.3 & 0.8140 & 0.8182 & -4.9 \\
\hline 30 & 0.8234 & 0.8236 & -0.3 & 0.8250 & -2.1 & 0.8291 & 0.8332 & -5.8 & 0.8314 & 0.8358 & -6.4 \\
\hline 40 & 0.8345 & 0.8353 & -1.2 & 0.8365 & -3.1 & 0.8408 & 0.8449 & -6.9 & 0.8434 & 0.8476 & -7.4 \\
\hline$\infty$ & 0.9 & 0.9 & - & 0.9 & - & 0.9 & 0.9 & - & 0.9 & 0.9 & - \\
\hline
\end{tabular}

the echelon stock-norms for the insertion department.

Each inserter may be modeled as a machine with two speeds, for instance $\nu_{\text {hor }}=0.23$ and 0 $\left(\times 10^{3} \mathrm{comp} . / \mathrm{min}\right.$. $)$ for the horizontal insert- ers. We suppose a $\nu_{\text {hor }}$-interval and a speed 0 interval are exponentially distributed with rates $\lambda=0.02875\left(=\frac{0.23}{8}\right)$ and $\mu=0.25875$, respec- 
TABLE 9

Parameters of insertion line and approximating line II

\begin{tabular}{|c|c|c|c|c|c|c|c|c|}
\hline$n=4$ & Prod. rate & Life rate & Trans. matrix & $E L+E M$ & $E T$ & $c_{\mathrm{T}}$ & $E S$ & $c_{\mathrm{s}}$ \\
\hline Line I & $\begin{array}{l}\mu_{1}=0.69 \\
\nu_{2}=0.46 \\
\nu_{3}=0.23 \\
\nu_{4}=0 \\
o_{1}=0.65 \\
o_{2}=0.34\end{array}$ & $\begin{array}{l}\lambda_{1}=0.086 \\
\lambda_{2}=0.316 \\
\lambda_{3}=0.546 \\
\lambda_{4}=0.776 \\
\mu_{1}=0.027 \\
\mu_{2}=0.248\end{array}$ & {$\left[\begin{array}{cccc}0 & 1 & 0 & 0 \\
0.82 & 0 & 0.18 & 0 \\
0 & 0.95 & 0 & 0.05 \\
0 & 0 & 1 & 0\end{array}\right.$} & 15.63 & 0.85 & 1.47 & 0.82 & 1.20 \\
\hline Line II & $\begin{array}{l}w_{1}=0.69 \\
w_{2}=0.41\end{array}$ & $\begin{array}{l}\rho_{1}=0.048 \\
\rho_{2}=0.350 \\
\eta_{1}=0.177 \\
\eta_{2}=0.482\end{array}$ & $\begin{array}{l}p=0.5 \\
q=0.5\end{array}$ & 15.63 & & & & \\
\hline
\end{tabular}

TABLE 10

Results for the insertion line

\begin{tabular}{rl}
\hline$K$ & $\tilde{V}_{\mathrm{I}}(K)\left(=V_{\mathrm{II}}(K)\right)$ \\
\hline 0 & 0.5666 \\
2 & 0.5991 \\
6 & 0.6098 \\
10 & 0.6133 \\
15 & 0.6153 \\
20 & 0.6164 \\
30 & 0.6175 \\
$\infty$ & 0.6192 \\
\hline
\end{tabular}

tively. $\mu=0.25875$ stems from the fact that the average downtime per inserter $\left(=\frac{\lambda}{\lambda+\mu}\right)$ is about $10 \%$ (see the Introduction). The three horizontal inserters are now equivalent to a single horizontal insertion $\mathrm{PU}$ with four different speeds $(0.69,0.46,0.23$ and 0$)$. A speed 0.46 -interval, for example, has an exponential distribution with rate $2 \lambda+\mu=0.31625$, since only two inserters are operating. The transition matrix is also easy to calculate. The same can be done for the vertical inserters, but for reasons of convenience, instead of modeling the insertion line as a line of Fig. 2 we have condensed the number of states of the vertical insertion PU to just two, both with exponential distribution. These states correspond to the situations where at least three, or less than three, inserters are operative, respectively. This is not serious since we have just shown that not the type of model is important, but only the type of buffer fluctuations. The resulting model is a line similar to the one in Fig. 3 (but with $\left.\omega_{2} \neq 0\right)$ and is presented in Table 9.

The speed of the second PU in line II is not equal to 1 (as in Fig. 5), but is 0.6192 , which equals the net production rate of the vertical insertion PU (the net production rate of the horizontal insertion PU is 0.621 ). In Table 10 the resulting throughouts $\hat{\nu}_{1}(K)$ are given.

Now suppose the desired throughput is about 613 (inserted) horizontal components per minute, that is about 8 printed circuits per minute. Then, in order to realise this throughput the total echelon stock in the insertion line must be kept equal to about 10,000 horizontal components at all times, that is to about 16 minutes of work for the vertical insertion PU.

Hence, the finiteness of the buffer capacity does not follow from a physical limitation for the storage of components, but by the way the system is controlled.

\section{ACKNOWLEDGEMENT}

Research supported by The Netherlands Organization for the Advancement of Pure Research (ZWO). 


\section{REFERENCES}

1 Wijngaard, J. (1987). Production control in a consumer electronics factory. J. Engineering Costs and Production Economics, 12(1-4): 165-173.

2 Van Donselaar, K. and Wijngaard, J. (1987). Commonality and safety stocks. J. Engineering Costs and Production Economics, 12(1-4): 165-173.

3 Wortmann, J.C. (1987). Information systems for assemble-to-order production. J. Engineering Costs and Production Economics, 12(1-4): 187-196.

4 Wijngaard, J. (1979). The effect of interstage buffer storage on the output of two unreliable production units in series, with different production rates. AIIE Transactions, 11: 42-47.

5 De Koster, M.B.M. and Wijngaard, J. (1985). Approximations of two-stage production lines with intermediate buffer. Report BDK/ORS/85/05, Eindhoven University of Technology, The Netherlands.

6 Bolch, G. and Akyildiz, I.F. (1982). Analyse von Rechensystemen. Teubner, Stuttgart.

7 Sauer, C.H. and Chandy, K.M., (1981). Computer Systems Performance Modelling. Prentice Hall, Englewood Cliffs, NJ.

8 Altiok, T. (1985). On the phase-type approximations of general distributions. IIE Transactions, 17: 110-116. 